\title{
BARBIER'S THEOREM IN THE LOBACHEVSKI PLANE
}

\section{JAY P. FILLMORE ${ }^{1}$}

\begin{abstract}
In the Lobachevski plane, horocycles with the same center are geodesic parallels and are natural replacements for the lines used in defining the support function of a convex curve and the notion of constant width in the Euclidean plane. In this paper, analogs based on horocycles are obtained for Christoffel's formula, which expresses the radius of curvature of a convex curve in terms of its support function, and Barbier's theorem, which relates the length and width of a convex curve of constant width.
\end{abstract}

1. Introduction. Santaló [4], among others, has employed families of horocycles in the Lobachevski plane to obtain the analogs of theorems of Euclidean geometry which depend upon families of lines in the Euclidean plane.

In the Lobachevski plane, horocycles are orthogonal trajectories to families of asymptotically parallel geodesics. Horocycles which are orthogonal to the same family of asymptotically parallel geodesics are geodesic parallels and it is natural to base a notion of constant width on this fact.

In the Euclidean plane, Barbier's theorem states that all curves of constant width $B$ have the same circumference $\pi B[2$, p. 200]. The proof of this theorem employs the Christoffel formula $1 / \kappa=h+h^{\prime \prime}$ where $h$ is the support function in the direction $\theta$, primes denote differentiation with respect to $\theta$, and $\kappa$ is the curvature of the (convex) curve at the point of support.

In this note we describe the analogs of the Christoffel and Barbier formulas for closed curves in the Lobachevski plane which are horocycle convex: every horocycle has at most two points in common with the curve.

An alternate approach to constant width in the Lobachevski plane, based on geodesics rather than horocycles, is discussed in Santaló [3]. The author thanks the referee for calling his attention to this paper.

2. Christoffel's formula. Let $K=-k^{2}(k>0)$ be the Gaussian curvature of the Lobachevski plane. The absolute is a circle and may

Received by the editors March 31, 1969.

AMS Subject Classifications. Primary 5225, 5330.

Key Words and Phrases. Convex curves, constant width, support function.

${ }^{1}$ Research supported in part by National Science Foundation grants. 
be given a coordinate $\theta(0 \leqq \theta<2 \pi)$ such that for any point of the plane, geodesics from that point going to the absolute through the point $\theta$ give geodesic polar coordinates with $\theta$ as the angular coordinate.

Let $O$ be a fixed point of the plane, and let $C$ be a piecewise smooth horocycle convex curve. For every point $\theta$ on the absolute, there is a unique horocycle having center at $\theta$, having $C$ on the geodesically convex side, and meeting $C$ in one point. Let $h(\theta)$ denote the distance from $O$ to the horocycle along a geodesic emanating from $O$ going through the point $\theta$, and measured positively toward $\theta . h(\theta)$ is the support function of $C$.

The curve $C$ may be obtained knowing $h(\theta)$ since it is the envelope of a family of horocycles. It is thus possible to compute the geodesic curvature $\kappa$ of $C$ at the point corresponding to $\theta$ in terms of $h$ alone. This computation, sketched in paragraph 4 , is long but direct and yields:

$$
\frac{1}{\kappa}=\frac{\left(e^{k h}-e^{-k h}\right) / 2 k-(k / 2) e^{-k h}\left(h^{\prime}\right)^{2}+e^{-k h} h^{\prime \prime}}{\left(e^{k h}+e^{-k h}\right) / 2+\left(k^{2} / 2\right) e^{-k h}\left(h^{\prime}\right)^{2}-k e^{-k h} h^{\prime \prime}} .
$$

The term appearing in the numerator is $d s / d \theta$, the derivative of arc length.

Note that in the limit $k \rightarrow 0$, this formula gives the Christoffel formula in the Euclidean case.

3. Barbier's theorem. Let $C$ be horocycle convex and $h(\theta)$ its support function. The length of $C$ is given by

$$
L=\int_{0}^{2 \pi}\left(\frac{e^{k h}-e^{-k h}}{2 k}+e^{-k h} h^{\prime \prime}-\frac{k}{2} e^{-k h}\left(h^{\prime}\right)^{2}\right) d \theta .
$$

Let $\bar{h}$ be the support function for $C$ constructed as was $h$ but with $C$ on the geodesically concave side of the horocycle. Replacing $h$ by $\bar{h}$ in the integral gives again the length of $C$, but with a change of sign.

The condition that $C$ be of constant width $B$ is expressed by $\bar{h}$ $=h-B$. From the two expressions for $L$ we obtain:

THEOREM. The length of a horocycle convex curve $C$ of constant width $B$ is given by

$$
L=\frac{1-e^{-k B}}{2 k} \int_{0}^{2 \pi} e^{k h} d \theta .
$$

Note that in the limit $k \rightarrow 0$ we obtain Barbier's theorem.

In the Lobachevski plane, not all curves of the same constant width have the same arc length. For example, the geodesic circle of constant 
width $B$ has length $L=2 \pi(\sinh (k B / 2) / k)$, while a Reuleux triangle of constant width $B$ has length $L=6(\sinh k B / k) \arcsin \left(\frac{1}{2} \operatorname{sech}(k B / 2)\right)$.

4. Computations. We work in Weierstrass coordinates $x, y, z$ $[5$, p. 74] $-\infty<x, y<\infty, z>1 / k$. These coordinates are related by $x^{2}+y^{2}-z^{2}=-1 / k^{2}$. The Riemannian metric is $d x^{2}+d y^{2}-d z^{2}$; the distance $d$ between the points having coordinates $(x, y, z)$ and $\left(x_{0}, y_{0}, z_{0}\right)$ is given by $\cosh k d=-k^{2}\left(x x_{0}+y y_{0}-z z_{0}\right)$. In terms of Weierstrass coordinates, geodesics, geodesic circles, and horocycles are described by linear equations. The absolute is coordinatized as described in 2.

The parametric equations

$$
\begin{aligned}
& x=e^{-k s} x_{0}+\sinh k s \cos \theta / k^{2}\left(z_{0}-x_{0} \cos \theta-y_{0} \sin \theta\right), \\
& y=e^{-k s} y_{0}+\sinh k s \sin \theta / k^{2}\left(z_{0}-x_{0} \cos \theta-y_{0} \sin \theta\right), \\
& z=e^{-k s} z_{0}+\sinh k s / k^{2}\left(z_{0}-x_{0} \cos \theta-y_{0} \sin \theta\right)
\end{aligned}
$$

describe the geodesic from the point with coordinates $\left(x_{0}, y_{0}, z_{0}\right)$ going through the point on the absolute with coordinate $\theta$ as arc length $s \rightarrow+\infty$. A fixed value of $\theta$ gives rise to a family of asymptotically parallel geodesics.

The geodesic circle with center $\left(x^{\prime}, y^{\prime}, z^{\prime}\right)$ going through $\left(x_{0}, y_{0}, z_{0}\right)$ is described by $-k^{2}\left(x^{\prime} x+y^{\prime} y-z^{\prime} z\right)=-k^{2}\left(x^{\prime} x_{0}+y^{\prime} y_{0}-z^{\prime} z_{0}\right)$. As $\left(x^{\prime}, y^{\prime}, z^{\prime}\right)$ tends to the point $\theta$ on the absolute, this geodesic circle tends to the horocycle with center $\theta$ passing through $\left(x_{0}, y_{0}, z_{0}\right)$. Its equation is $\left(x-x_{0}\right) \cos \theta+\left(y-y_{0}\right) \sin \theta-\left(z-z_{0}\right)=0$. For fixed $\theta$, and various choices of $C$, the equations $x \cos \theta+y \sin \theta-z=C$ represent parallel horocycles. These are geodesic parallels and any two such cut off segments of the same length on any geodesic through $\theta$. For the choices $C$ and $C^{\prime}$, the length of this segment is $(1 / k)\left|\log \left(C^{\prime} / C\right)\right|$.

Let $O$ have coordinates $x=0, y=0, z=1 / k$. From the above considerations, the equation $x \cos \theta+y \sin \theta-z=-(1 / k) e^{-k h}$ represents the horocycle having center $\theta$ on the absolute and having distance $h$ from $O, h$ being measured positive toward $\theta$.

Let $h=h(\theta)$ be the support function of a piecewise smooth horocycle convex curve $C$. Then $C$ is the envelope of the horocycles whose equations are

$$
x \cos \theta+y \sin \theta-z=-(1 / k) e^{-k h(\theta)} .
$$

The equations of $C, x, y$, and $z$ as functions of $\theta$, are obtained by solving this equation simultaneously with its derivative with respect to 
$\theta:-x \sin \theta+y \cos \theta=e^{-k h} h^{\prime}$, and the relation between the coordinates: $x^{2}+y^{2}-z^{2}=-1 / k^{2}$. They are

$$
\begin{aligned}
& x=\left(\frac{1}{2 k} e^{k h}+\left(\frac{k}{2}\left(h^{\prime}\right)^{2}-\frac{1}{2 k}\right) e^{-k h}\right) \cos \theta-e^{-k h} h^{\prime} \sin \theta, \\
& y=\left(\frac{1}{2 k} e^{k h}+\left(\frac{k}{2}\left(h^{\prime}\right)^{2}-\frac{1}{2 k}\right) e^{-k h}\right) \sin \theta+e^{-k h} h^{\prime} \cos \theta \\
& z=\frac{1}{2 k} e^{k h}+\left(\frac{k}{2}\left(h^{\prime}\right)^{2}+\frac{1}{2 k}\right) e^{-k h} .
\end{aligned}
$$

The derivative of arc length with respect to $\theta, d s / d \theta$, is now computed as the square root of $(d x / d \theta)^{2}+(d y / d \theta)^{2}-(d z / d \theta)^{2}$. One obtains the numerator in the expression for $1 / \kappa$ in 2 .

The unit tangent to $C$ at the point of support for the horocycle with center $\theta$ has components

$$
\begin{aligned}
u & =k h^{\prime} \cos \theta-\sin \theta, \\
v & =k h^{\prime} \sin \theta+\cos \theta, \\
w & =k h^{\prime} ;
\end{aligned}
$$

and the unit outward normal to $C$ at the same point has components

$$
\begin{aligned}
& l=\left(-\frac{e^{k h}+e^{-k h}}{2}+\frac{k^{2}}{2} e^{-k h}\left(h^{\prime}\right)^{2}\right) \cos \theta-k e^{-k h} h^{\prime} \sin \theta, \\
& m=\left(-\frac{e^{k h}+e^{-k h}}{2}+\frac{k^{2}}{2} e^{-k h}\left(h^{\prime}\right)^{2}\right) \sin \theta+k e^{-k h} h^{\prime} \cos \theta, \\
& n=-\frac{e^{k h}-e^{-k h}}{2}+\frac{k^{2}}{2} e^{-k h}\left(h^{\prime}\right)^{2} .
\end{aligned}
$$

The normal is obtained by simultaneously solving the equations $l^{2}+m^{2}-n^{2}=1, u l+v m-w n=0$, and $x l+y m-z n=0$.

The geodesic curvature $\kappa$ is the component along the unit normal of the intrinsic derivative of the unit tangent with respect to $s$. This turns out to equal $l d u / d s+m d v / d s-n d w / d s$. Hence $\kappa d s / d \theta=l d u / d \theta$ $+m d v / d \theta-n d w / d \theta$ and for this one obtains the denominator in the expression for $1 / \kappa$ in 2 .

To obtain the formula of the theorem in 3, replace $h$ by $\bar{h}=h-B$ in the first expression for $L$ to obtain an expression for $-L$. Factoring out $e^{h B}$ and using the original expression for $L$ gives 


$$
L=-e^{k B} L+\frac{e^{k B}-e^{-k B}}{2 k} \int_{0}^{2 \pi} e^{k h} d \theta
$$

from which the theorem follows.

5. Concluding remark. The expression in 2 for $1 / \kappa$ may be interpreted as a differential equation for $h$ given the radius $1 / \kappa$ of the osculating Euclidean circle. If instead we use radius $R$ of the osculating geodesic circle, related to the former radius by $\tanh k R / k=1 / \kappa$, we obtain

$$
R=h-(1 / 2 k) \log \left(1+k^{2}\left(h^{\prime}\right)^{2}-2 k h^{\prime \prime}\right) .
$$

This yields an algebraic differential equation for $h$ given $R^{\prime}$. An explicit solution to either of these differential equations and their higher dimensional analogs based on horospheres would permit a study of the connection between convexity and principle radii of curvature along the lines of the Euclidean case initiated by Christoffel and completed by Firey [1].

\section{REFERENCES}

1. W. J. Firey, The determination of convex bodies from their mean radius of curvature functions, Mathematika 14 (1967), 1-13. MR 36 \#788.

2. D. Laugwitz, Differential and Riemannian geometry, Academic Press, New York, 1965. MR 30 \#2406.

3. L. A. Santalo, Note on convex curves on the hyperbolic plane, Bull. Amer. Math. Soc. 51 (1945), 405-412. MR 7, 26.

4. - Horocycles and convex sets in hyperbolic plane, Arch. Math. (Basel) 18 (1967), 529-533. MR 37 \#870.

5. P. A. Sirokov, A sketch of the fundamentals of Lobachevskian geometry, Noordhoff, Groningen, 1964. MR 28 \#4419.

University of California at San Diego 